\title{
High-resolution fast low-angle shot magnetic resonance imaging of the normal hand
}

\author{
Harald Bruhn, M.D., Ph.D., Michael L. Gyngell, Ph.D., Wolfgang Hänicke, Dipl. Math., \\ Klaus-Dietmar Merboldt, Ph.D., and Jens Frahm, Ph.D.
}

Max-Planck Institute for Biophysical Chemistry, Göttingen, FRG

\begin{abstract}
Cross-sectional magnetic resonance (MR) images of the normal hand, wrist, and fingers with an inplane resolution of $0.2-0.4 \mathrm{~mm}$ and a slice thickness of 1-2 $\mathrm{mm}$ were obtained using a $40-\mathrm{cm}$ bore, $2.35-\mathrm{T}$ MRI system equipped with actively shielded $50 \mathrm{mT} \mathrm{m}^{-1}$ gradient coils. A detailed description of the normal anatomy is given. The $\mathrm{T}_{1}$-weighted, multi-slice, fast low-angle shot (FLASH) MR images presented show a substantial improvement in resolution as compared with earlier reports. Typical investigational times of about 15 min offer a fast scan protocol that is suitable for routine clinical applications. The study further demonstrates the potential of dedicated magnets to facilitate and refine diagnostic MR imaging of hand injuries and hand-related diseases.
\end{abstract}

Key words: Magnetic resonance (MR) imaging - Joints, MR studies - Wrist, MR studies - Hand, MR studiesFinger, MR studies - Wrist anatomy

Magnetic resonance imaging (MRI) of the hand aims to demonstrate anatomical structures and pathologies in the greatest possible detail $[1-3,6-10,12-14,16,18-$ 20]. Unfortunately, the spatial resolution obtained up to now has been limited by the relatively weak gradient strengths available with conventional whole-body MRI systems. As a consequence, partial volume effects lead to blurring of structural details and thus tend to impair diagnostic accuracy and certainty. Moreover, when whole-body magnets are employed the patient is subjected to an uncomfortable and/or off-center position.

This study reports on the use of a MRI system comprising a small-bore, high-field magnet with a strong magnetic field gradient system. This combination allows the acquisition of high-resolution MR images that sur-

Address reprint requests to: Harald Bruhn, M.D., Ph.D., MaxPlanck-Institut für biophysikalische Chemie, Postfach 2841, W3400 Göttingen, FRG pass the quality of those obtained with whole-body magnets. Subjects may sit or lie in front of the magnet with the arm extended into the tunnel. Representative crosssectional images of the normal human hand, wrist, and fingers have been selected from multislice acquisitions for their value in depicting typical anatomical structures.

\section{Subjects and methods}

All MRI investigations were performed at $2.35 \mathrm{~T}$ using a $40-\mathrm{cm}$ bore horizontal magnet (Bruker) equipped with an actively shielded gradient system (Oxford Instruments). The maximum available gradient strength was $50 \mathrm{mT} \mathrm{m}^{-1}$. Radio frequency (RF) excitation and detection were accomplished using a 10.5-cm diameter birdcage RF coil for hand and wrist imaging and a 4-cm diameter Helmholtz RF coil for finger studies, respectively. Fast scan, $\mathrm{T}_{1^{*}}$ weighted, multislice, fast low-angle shot (FLASH) MR images [5] were acquired with a repetition time of $150 \mathrm{~ms}$ and a flip angle of $70^{\circ}$. In most cases an echo time (TE) of $7.15 \mathrm{~ms}$ was chosen to achieve "opposed phase" conditions for fat and water signals. Under these circumstances, fat and water signals mutually cancel out if they contribute to the same image pixel, resulting in superior image contrast. Typically, nine slices each with a thickness of 2 $\mathrm{mm}(1 \mathrm{~mm}$ for fingers) and a center-to-center distance of $2.5 \mathrm{~mm}$ $(1.25 \mathrm{~mm}$ for fingers) were obtained. The total measuring time amounted to about $2.5 \mathrm{~min}$, with four accumulations for signal-tonoise improvements. If not otherwise stated in the figure captions, the images represent a field of view (FOV) of $100 \mathrm{~mm}(50 \mathrm{~mm}$ for fingers) that is covered by a data matrix of $256 \times 256$ pixels yielding linear pixel dimensions of $0.2-0.4 \mathrm{~mm}$. A few images were also acquired with an image matrix of $512 \times 512$ pixels with a $0.2-$ $\mathrm{mm}$ resolution.

Multislice images were obtained in transverse, coronal, and sagittal orientation from the wrists, hands, and fingers of 15 asymptomatic volunteers who were $20-30$ years old. Informed consent was obtained prior to the investigation. A typical protocol for complete wrist studies included two sets of transverse and coronal acquisitions yielding two sets of 18 multiplanar images. Sagittal views provided no additional information and were made in only a few cases. The volunteers were positioned either sitting or lying with an arm extended into the magnet, which had an isocenter $57 \mathrm{~cm}$ from the face of the magnet. While this posed no difficulty for the volunteers, a short arm length would obviously exclude many patients from investigation. However, the design of a dedicated system with a shorter magnet is possible and would eliminate this problem. 

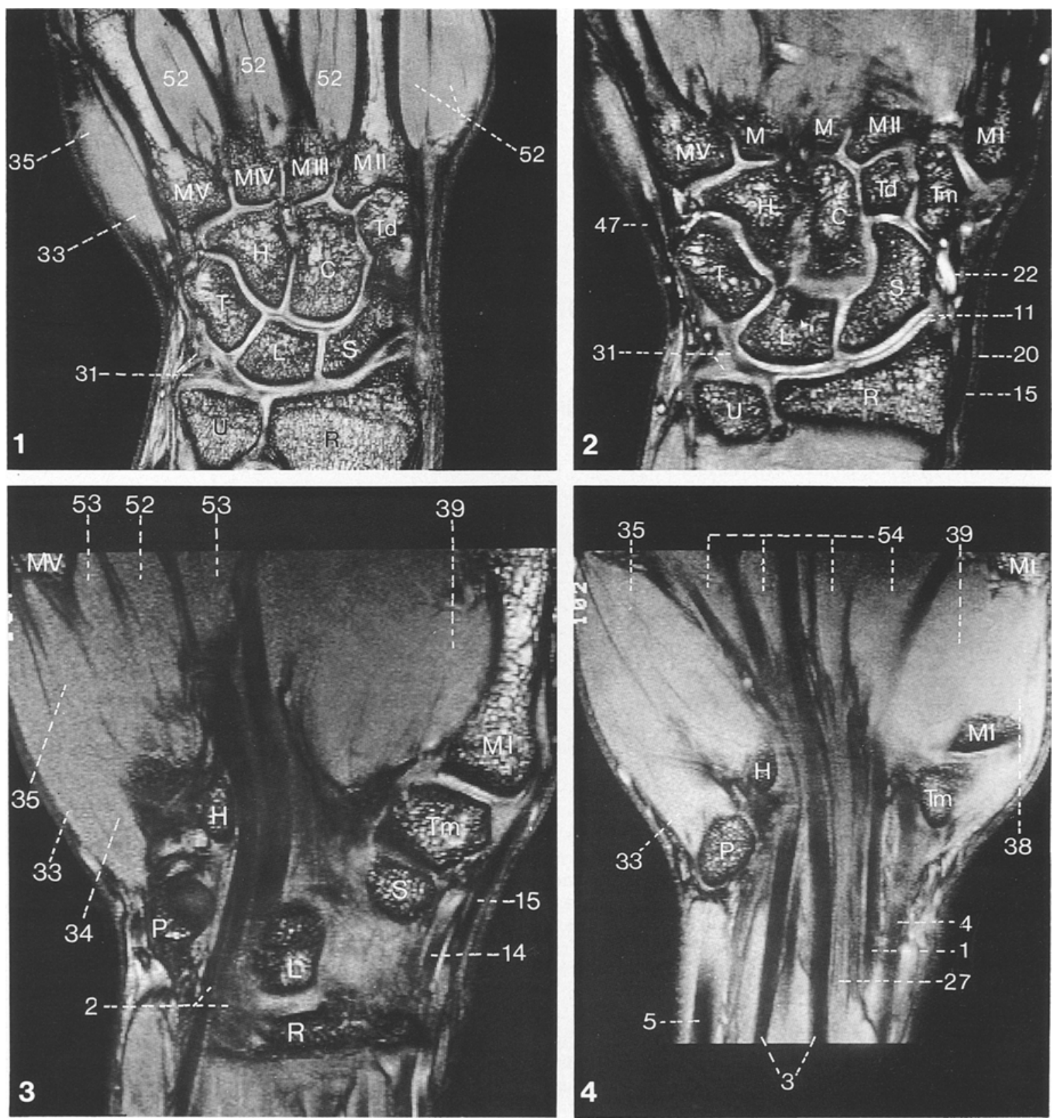

Fig. 1. Coronal view of the wrist in the plane of bones and joints. The following bones are identified: the distal part of radius and ulna, scaphoid, lunate, triquetral, hamate, capitate, trapezoid, and the second to fifth metacarpals. The hyperintense articular cartilage in the radioulnar, radiocarpal, intercarpal, and carpometacarpal joints is clearly discerned. Its smooth surface is best seen when the low-signal synovial cavity can be resolved. At the radiocarpal joint, parts of the dorsal radiocarpal and transverse intercarpal ligaments are depicted, showing low signal intensity. This also applies to the dorsal aspect of the triangular fibrocartilage complex at the ulnocarpal transition with its ulnar attachment and ulnar collateral ligament. The strong interosseous capitohamate ligament can be seen. Between the metacarpals the interosseous muscles are sectioned, and it is clear how the contiguous bases of these bones are connected by interosseous ligaments, just distal to their collateral articular facets

Fig. 2. Coronal image volar to Fig. 1. The distal ends of radius and ulna, the carpal bones, and the first and fifth metacarpals are sectioned. The fixation and extension of the triangular fibrocartilage complex is shown with low signal intensities of different degrees. The intercarpal ligaments, e.g., the scapholunate, the lunatotriquetral (connecting transversely the bones of the first carpal row), the interosseous ligaments (connecting the bones of the distal row with each other, with the first row, and with the metacarpals), and the collateral radial and uinar ligaments are clearly differentiated from the hyperintense hyaline articular cartilage. The extensor pollicis tendon is observed radially. A chance finding in this particular volunteer is a round area of signal loss in the lunate (marked by arrowhead) due to calcification.

Fig. 3. Coronal view deep in the carpal tunnel. The pisiform and hook of hamate form the carpal tunnel on the hypothenar side, and the tubercles of the scaphoid and trapezium on the thenar side. The course of two flexor digitorum profundus tendons is displayed. Muscles of the hypothenar eminence and the adductor pollicis muscle are sectioned

Fig. 4. Coronal view volar to Fig. 3. The flexor digitorum superficialis, flexor pollicis, and flexor carpi tendons are seen. The course of the median nerve is shown as it branches distally at the level of the proximal metacarpus. The flexor carpi tendons are partially sectioned 
Table 1. List of anatomical assignments

$\mathrm{R}=$ radius (bone, marrow), $\mathrm{U}=$ ulna (bone, marrow), $\mathrm{S}=$ scaphoid, $\mathrm{L}=$ lunate, $\mathrm{T}=$ triquetral, $\mathrm{P}=$ pisiform, $\mathrm{H}=$ hamate, $\mathrm{C}=$ capitate, $\mathrm{Td}=$ trapezoid, $\mathrm{Tm}=$ trapezium, $\mathrm{M}=$ metacarpal, $\mathrm{Ph}=$ phalanx

$1=$ flexor pollicis longus muscle and tendon, $2=$ flexor digitorum profundus muscle and tendons, $3=$ flexor digitorum superficialis muscle and tendons, $4=$ flexor carpi radialis muscle and tendon, $5=$ flexor carpi ulnaris muscle and tendon, $6=$ extensor pollicis longus muscle and tendon, $7=$ extensor digitorum muscle and tendons, $8=$ extensor indicis muscle and tendon, $9=$ extensor digiti minimi muscle and tendon, $10=$ extensor carpi ulnaris muscle and tendon, $11=$ articular cartilage, articular capsule, $12=$ interosseous artery, nerve and vein, 13 =brachioradialis tendon, $14=$ abductor pollicis longus muscle and tendon, $15=$ extensor pollicis brevis muscle and tendon, $16=$ extensor carpi radialis brevis muscle and tendon, $17=$ extensor carpi radialis longus muscle and tendon, $18=$ pronator quadratus muscle, $19=$ palmaris longus muscle and tendon, $20=$ antebrachial fascia, $21=$ dermis, $22=$ radial artery, $23=$ cephalic vein, $24=$ ulnar artery, $25=$ ulnar nerve, $26=$ basilic vein, $27=$ median nerve, $28=$ intermuscular septum, $29=$ flexor retinaculum, $30=$ extensor retinaculum, $31=$ triangular articular meniscus, triangular fibrocartilage complex, $32=$ synovial sheath

Muscles of the hypothenar eminence: $33=$ abductor digiti minimi muscle, $34=$ flexor digiti minimi brevis muscle, $35=$ opponens digiti minimi muscle

Muscles of the thenar eminence: $36=$ abductor pollicis brevis muscle, $37=$ flexor pollicis brevis muscle, $38=$ opponens pollicis muscle,
$39=$ adductor pollicis muscle, $40=$ deep branch of ulnar artery, vein, and nerve; deep palmar arch

Intrinsic (intracapsular) ligaments: $41=$ dorsal radiocarpal; radiolunatotriquetral; $42=$ dorsal intercarpal; interosseous ligaments: scapholunate, lunatotriquetral, capitohamate, capitotrapezoid, trapezoidotrapezium; 43 = palmar radiocarpal; radioscaphoid (RSL), radiotriquetral (RTL), radiocapitate (RCL); 44= palmar ulnocarpal; ulnolunate (ULL), ulnotriquetral (UTL), 45 = radiate carpal ligament and palmar intercarpal; capitotriquetral, capitolunate, and capitoscaphoid; pisohamate ligament

Extrinsic (capsular) ligaments: $46=$ radial collateral ligament, $47=$ ulnar collateral ligament, $48=$ dorsal carpometacarpal ligament, $49=$ palmar carpometacarpal ligament, $50=$ palmar brevis muscle, $51=$ palmar aponeurosis, $52=$ dorsal interosseous muscle, $53=$ palmar interosseous muscle, $54=$ lumbrical muscle, $55=$ dorsal metacarpal ligament, $56=$ palmar metacarpal ligament

Anatomical structures of the finger: $\mathrm{P}_{p}=$ proximal phalanx, $\mathrm{P}_{m}=$ middle phalanx, $\mathrm{P}_{d}=$ distal phalanx

$60=$ volar plate, $61=$ articular cartilage, $62=$ flexor digitorum ind cis profundus tendon, $63=$ flexor digitorum indicis superficialis tendon, $64=$ collateral tendinous insertion of lumbrical and interosseous muscles into extensor expansion; radial callosity, $65=$ palmar digital artery and nerve, $66=$ dorsal digital artery and nerve, $67=$ digital tendon sheath (vagina synovialis tendinis), $68=$ extensor digitorum indicis tendon and expansion, $69=$ ungual bed

\section{Results and discussion}

Multislice MR images as typically recorded from distal forearm to finger were selected for display with respect to their representative value in depicting anatomical structures and in demonstrating the resolution achieved. They are organized according to scan orientation: (a) Figs. 1-4, coronal sections of the wrist from dorsal to palmar, (b) Figs. 5-10, transverse sections from the distal forearm to the metacarpus, (c) Figs. 11-16, sagittal sections of the wrist from the radial to ulnar side, and (d) Figs. 17 and 18, sagittal and transverse sections of the finger. The hand has been positioned with a slight dorsal flexion. In the coronal and transverse MR images the radius and thenar eminence are displayed to the viewer's right. Features of anatomy were identified by referring to recognized texts of hand anatomy $[4,11$, $15,17]$. Structural details in the images are indicated by numbers and letters.

This study demonstrates marked improvements in the use of MRI to study the wrist, hand, and fingers. The present achievements are based on a substantial increase in image resolution that can be obtained in a comfortable investigation and within relatively short measuring times by means of multislice FLASH MRI studies on a small-bore magnet. In most cases, images with a slice thickness of $2 \mathrm{~mm}$, a data matrix of $256 \times 256$ pixels, and a FOV of $75-100 \mathrm{~mm}$ turned out to be adequate for routine hand studies. A further improvement of the spatial resolution by an increase of the data matrix to $512 \times 512$ pixels or a reduction of the slice thickness to $1 \mathrm{~mm}$ did not balance either the resulting loss in signalto-noise ratio or the increase in measuring time.

The present results illustrate the potential of a smallbore, high-field, MRI system as a superior tool for pertinent diagnostic applications in a hand clinic. The design of shorter magnets will further improve the convenience of such studies and guarantee general applicability. MRI applications will extend into three-dimensional imaging. In fact, the delineation of the complex anatomy encountered in the hand will benefit from the accessible isotropic resolution as well as from the capability for reconstruction to create selected views in the form of arbitrary sections and curved inner surfaces.

Acknowledgements. Financial support by the Bundesminister für Forschung und Technologie (BMFT) of the Federal Republic of Germany (grant 01 VF 8606/6) is gratefully acknowledged. Special thanks are extended to Heide Birkefeld and Michaela Beusse for preparation of the photographs. 

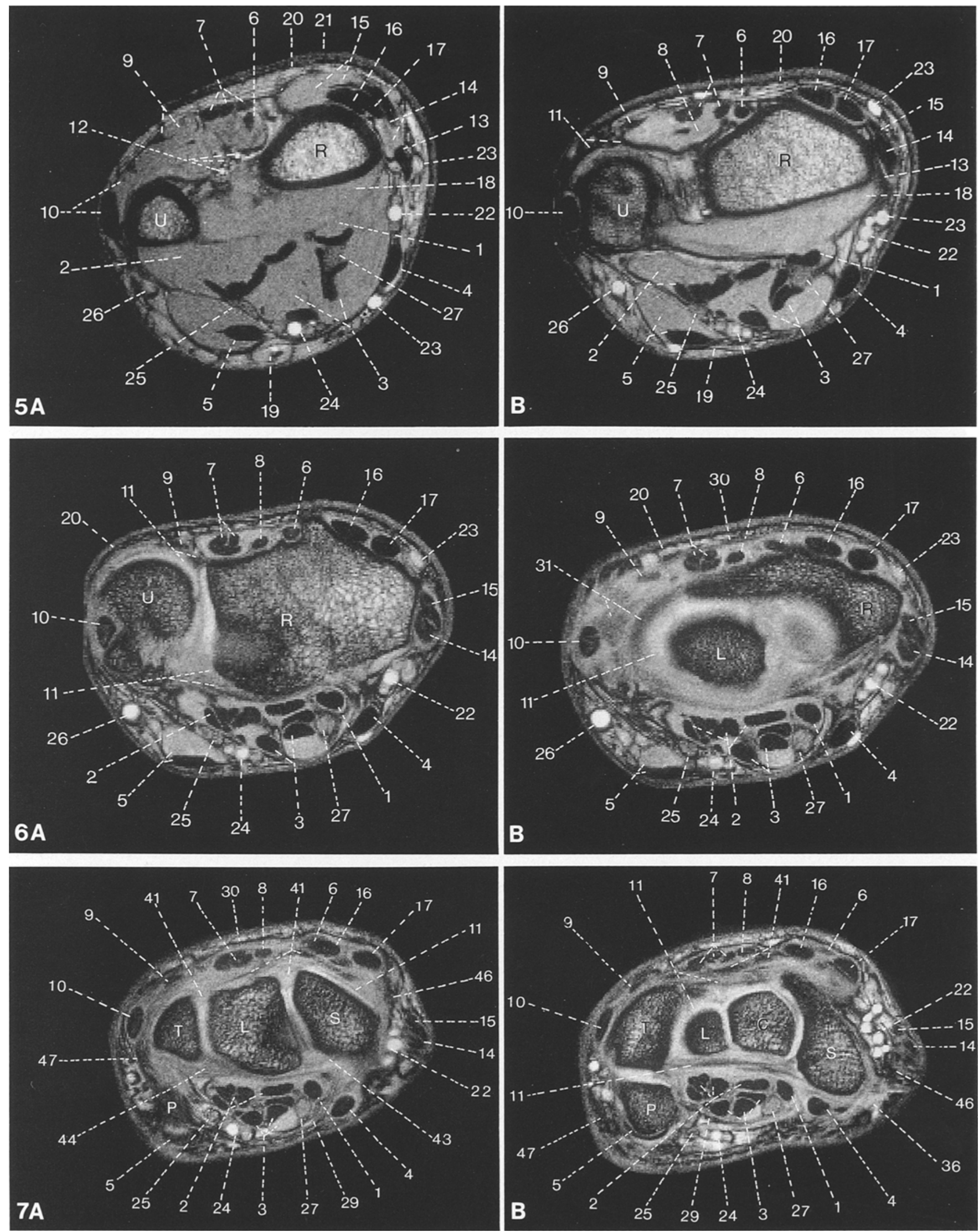

Figs. 5-7. See next page for corresponding captions 

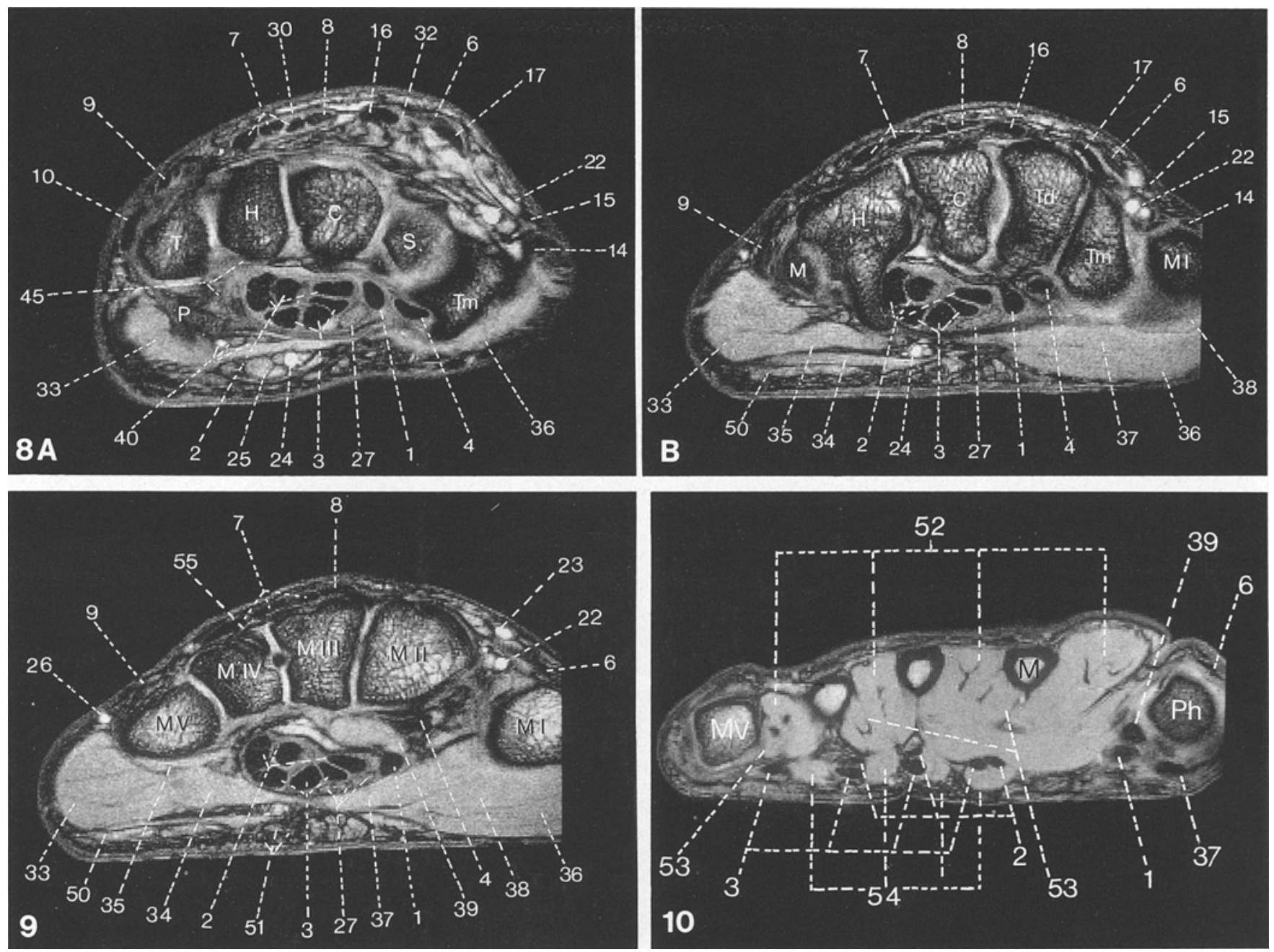

Fig. 5A, B. Transverse views of distal quarter of forearm. A Proximal section: the origin and course of flexor and extensor tendons attached to their muscle bellies are demonstrated. Cross-sections of the median and ulnar nerves are well delineated by a dotted appearance hypointense to muscle tissue. Vessels with flowing blood appear bright. B Section distal to $\mathbf{A}$ : the transverse extension of the pronator quadratus muscle is shown as it originates from the anterior margin of the ulna and inserts at the anterior face of the radius. The radioulnar joint is sectioned proximally at the sacciform recess

Fig. 6A, B. Transverse views at the transition of foream to wrist. A Radioulnar joint: the thickness and surface of the articular cartilage can be assessed. Most tendons have left their muscle bellies behind and are surrounded by their synovial sheaths. Between ulnar styloid and medial concavity of the radius the articular disc commences to extend as part of the triangular fibrocartilage complex. The dorsal and volar radioulnar ligaments strengthen the fibrous articular capsule. B Radiocarpal joint: additional parts of the fibrocartilage complex appearing hypointense are sectioned, e.g., the ulnocarpal meniscus, collateral ulnar ligament, and palmar ulnocarpal ligaments. The hyaline articular cartilage that extends around the lunate head and the synovial sheaths of the tendons appear hyperintense

Fig. 7A, B. Transverse views in the proximal row of carpal bones. A Scaphoid, lunate, and triquetral are sectioned. Their transverse ligamentous connections are appreciated by signal intensities lower than hyaline articular cartilage. The bones of the wrist continue the dorsal roof of the carpal tunnel. Its volar encasement by the flexor retinaculum

and its contents, e.g., flexor tendons and median nerve, can be discerned clearly. The synovial tendon sheaths are distinguished by their bright appearances. Fibrous structures including the articular capsule, carpal ligaments, and extensor and flexor retinaculum are observed as hypointensities. B Section distal to A. The enclosing structures of the carpal tunnel are well delineated between pisiform and scaphoid tubercle

Fig. 8A, B. Transverse views in the distal row of carpal bones. A Pisiform, scaphoid, and triquetral are still identifiable, while the lunate has given way to hamate and capitate. The radial flexor carpi is observed as it runs through the groove of the trapezium. The muscles of the thenar and hypothenar eminences start their course. B Trapezoid, trapezium, capitate, hamate, first and fifth metacarpal heads are sectioned. The hook of the hamate forms the lateral limitation of the carpal tunnel on the hypothenar side. The transverse cross-section of the median nerve becomes flat and starts to branch

Fig. 9. Transverse section at the level of the proximal metacarpus. The distal carpal bones have disappeared, and the five metacarpal heads are sectioned. The adductor pollicis muscle interfaces carpal tunnel and bones. The bundle of flexor tendons is about to flatten, and the median nerve can be discerned as its branches diverge

Fig. 10. Transverse view of the metacarpus at the level of the first and fifth metacarpophalangeal joints. Dorsal and palmar interosseous as well as lumbrical muscles are cross-sectioned. Flexor digitorum profundus and superficialis tendons can readily be discerned as they run towards their digits 

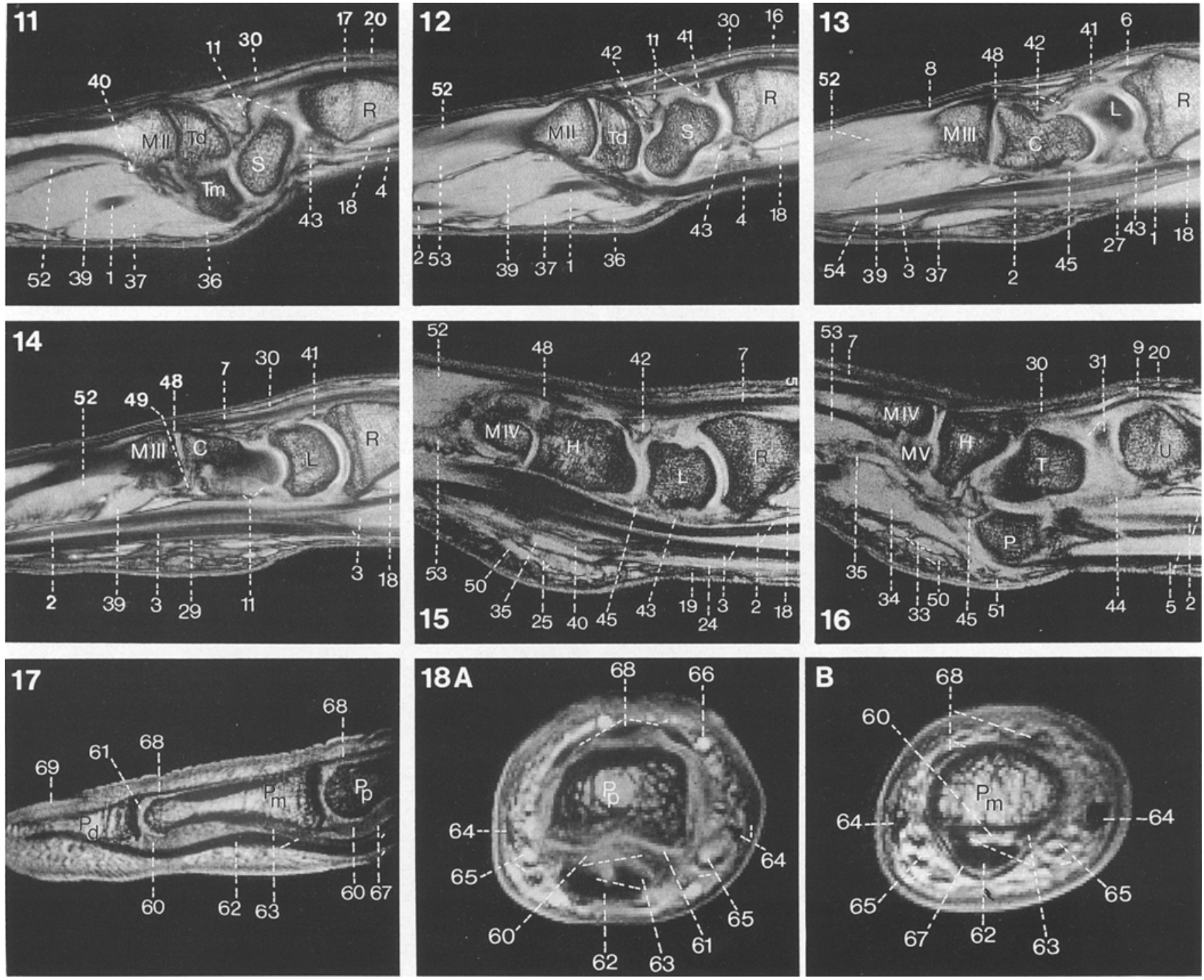

Fig. 11. Sagittal view of the radial wrist. The distal radius, scaphoid, trapezium, trapezoid, and second metacarpal bones are sectioned

Fig. 12. Sagittal section medial of Fig. 11. The distal course of the radial flexor carpi is observed running below the scaphoid and bending upwards through its groove at the trapezium to reach finally its insertion at the base of the second metacarpal bone

Fig. 13. Sagittal view of the central wrist. The capitate is sectioned approximately in the midline and the lunate and third metacarpal bases, radially. The median nerve is shown as it travels distally beneath the flexor pollicis longus tendon and a flexor profundus tendon. Dorsal and palmar radiocarpal, dorsal carpometacarpal, and radiate ligaments are identified in respective parts

Fig. 14. Sagittal section ulnar to Fig. 13. The radial vertical concavity fitting the proximal lunate convexity is demonstrated. The capitate and the base of the third metacarpal are sectioned at their ulnar aspect. The course of extensor and flexor profundus and superficialis tendons running in hyperintense synovial sheaths is depicted

Fig. 15. Sagittal section through the distal radius, lunate, hamate, and medial base of the fourth metacarpal bone, ulnar to Fig. 14. The flexor profundus and superficialis tendons at the ulnar side of the carpal tunnel are noted as they pass by the medial convex cavity of the hook of the hamate. Its tip is observed as a hypointensity immediately below the flexor superficialis tendon (note: $\mathrm{FOV}=7.5 \mathrm{~cm}$ )
Fig. 16. Sagittal view of the ulnar wrist. The distal ulnar end, triquetral pisiform, hamate, fourth metacarpal, and the base of the fifth metacarpal bone are displayed in their complex relationship. Between ulna and triquetral, palmar ulnocarpal, and dorsal radiocarpal ligaments the small fibrocartilaginous meniscus is identified. The distal course of the ulnar flexor carpi inserting at the pisohamate is depicted (note: $\mathrm{FOV}=7.5 \mathrm{~cm}$ )

Fig. 17. Sagittal view of the central index finger. The course of the flexor profundus tendon under the phalanges and its insertion at the palmar surface of the distal phalanx are observed. It runs posterior to the flexor superficialis tendon, having pierced the latter anteriorly to the proximal phalanx. The attachment of the flexor superficialis is observed at the palmar surface of the base of the middle phalanx. The articular cartilage in the phalangeal joints and the extension of the volar plate can be assessed (note: $F O V=5.0 \mathrm{~cm}, \mathrm{TE}=5.7 \mathrm{~ms}$ )

Fig. 18A, B. Transverse views of the index finger. A The proximal phalanx is sectioned near the interphalangeal joint. The three fascicles of the extensor expansion are clearly depicted. The articular fibrous capsule is fortified by collateral ligaments. The flexor superficialis tendon has already diverged into halves. The osseoaponeurotic volar plate forms a concavity lined by a synovial sheath in which the flexor tendons are received. $\mathbf{B}$ The middle phalanx is sectioned. The digital fibrous sheath is appreciated as a low-signal arch that is attached to the margins of the phalanx and spans across the flexor tendons and their hyperintense synovial sheath. The middle fascicle of the extensor expansion inserts onto this phalanx (note: $F O V=5.0 \mathrm{~cm}, \mathrm{TE}=5.7 \mathrm{~ms}$ ) 


\section{References}

1. Baker LL, Hajek PC, Björkengren A, Galbraith R, Sartoris DJ, Gelberman RH, Resnick D (1987) High-resolution magnetic resonance imaging of the wrist: normal anatomy. Skeletal Radiol 16:128

2. Binkovitz LA, Cahill DR, Ehman RL, Berquist TH (1988) Magnetic resonance imaging of the wrist: normal cross sectional imaging and selected abnormal cases. Radio Graphics 8:1171

3. Erickson SJ, Kneeland BJ, Middleton WD, Jesmanowics A, Hyde J, Lawson TL, Foley WD (1989) MR imaging of the finger: correlation with normal anatomic sections. AJR 152:1013

4. Ferner H, Staubesand J (eds) (1976) Atlas der Anatomie des Menschen/Sobotta, 18th edn. Urban \& Schwarzenberg, Munich, p 327

5. Frahm J, Gyngell ML, Hänicke W (1991) Rapid scan techniques. In: Stark DD, Bradley WG (eds) Magnetic resonance imaging, 2nd edn., Mosby, St. Louis

6. Golimbu CN, Firooznia H, Melone CP, Rafii M, Weinreb J, Leber CL (1989) Tears of the triangular fibrocartilage of the wrist: MR imaging. Radiology 173:731

7. Gundry CR, Kursunoglu-Brahme S, Schwaighofer B, Kang HS, Sartoris DJ, Resnick D (1990) Is MR better than arthrography for evaluating the ligaments of the wrists? In vitro study. AJR 154:337

8. Hinshaw WS, Bottomley PA, Holland GN (1977) Radiographic thin-section imaging of the human wrist by nuclear magnetic imaging. Nature 270:722

9. Hinshaw WS, Andrew ER, Bottomley PA, Holland GN, Moore
WS, Worthington BS (1979) An in-vivo study of the forearm and hand by thin-section NMR imaging. Br J Radiol 52:36

10. König H, Lucas D, Meissner R (1986) The wrist: a preliminary report on high-resolution MR imaging. Radiology 160:463

11. Mayfield JK, Johnson RP, Kilcoyne RF (1976) The ligaments of the human wrist and their functional significance. Anat Rec $186: 417$

12. Mesgarzadeh M, Schneck CD, Bonakdarpour A (1989) Carpal tunnel: MR imaging part I. Normal anatomy. Radiology $171: 743$

13. Mesgarzadeh $M$, Schneck CD, Bonakdarpour A, Mitra A, Conaway D (1989) Carpal tunnel: MR imaging part II. Carpal tunnel syndrome. Radiology 171:749

14. Middleton WD, Lawson TL (1987) Anatomy and MRI of the joints. Raven Press, New York, p 83

15. Palmer AK, Werner FW (1981) The triangular fibrocartilage complex of the wrist - anatomy and function. J Hand Surg [Am] 6:153

16. Quinn SF, Belsole RJ, Greene TL, Rayhack JM (1989) Advanced imaging of the wrist. Radio Graphics 9:229

17. Warwick H, Williams PL (eds) (1973) Gray's anatomy, 3rd edn. Longman, Edinburgh; pp 432, 542, 651, 699

18. Weiss KL, Beltran J, Shaman O, Stilla RF, Levey M (1986) High-ficld MR surface-coil imaging of the hand and wrist. I. Normal anatomy, Radiology 160:143

19. Weiss KL, Beltran J, Lubbers LN (1986) High-field MR surface-coil imaging of the hand and wrist. II. Pathologic correlations and clinical relevance. Radiology 160:147

20. Zlatkin MB, Chao PC, Osterman AL, Schnall MD, Dalinka MK, Kressel HY (1989) Chronic wrist pain: evaluation with high-resolution MR imaging. Radiology 173:723 